Draft version OCtober 30, 2018

Preprint typeset using $\mathrm{LAT}_{\mathrm{E}} \mathrm{X}$ style emulateapj v. 08/22/09

\title{
ENHANCED PRODUCTION OF BARIUM IN LOW-MASS STARS: EVIDENCE FROM OPEN CLUSTERS
}

\author{
VALENTINA D'Orazi \\ Dipartimento di Astronomia e Scienza dello Spazio, Università di Firenze, Firenze, Italy and \\ INAF -Osservatorio Astrofisico di Arcetri, Firenze, Italy \\ Laura Magrini, Sofia Randich, and Daniele Galli \\ INAF -Osservatorio Astrofisico di Arcetri, Firenze, Italy \\ MAurizio Busso \\ Dipartimento di Fisica, Università di Perugia, and Sezione INFN, Perugia, Italy \\ PaOla Sestito \\ INAF - Osservatorio Astrofisico di Arcetri, Firenze, Italy \\ Draft version October 30, 2018
}

\begin{abstract}
We report the discovery of a trend of increasing barium abundance with decreasing age for a large sample of Galactic open clusters. The observed pattern of $[\mathrm{Ba} / \mathrm{Fe}]$ vs. age can be reproduced with a Galactic chemical evolution model only assuming a higher Ba yield from the $s$-process in low-mass stars than the average one suggested by parametrized models of neutron-capture nucleosynthesis. We show that this is possible in a scenario where the efficiency of the extra-mixing processes producing the neutron source ${ }^{13} \mathrm{C}$ is anti-correlated with the initial mass, with a larger efficiency for lower masses. This is similar to the known trend of extended mixing episodes acting in H-rich layers and might suggest a common physical mechanism.

Subject headings: Galaxy: disk, abundances, evolution; open clusters and associations: general, abundances
\end{abstract}

\section{INTRODUCTION}

Barium is one of the so-called heavy neutron-capture elements. It is formed by neutron captures occurring through two major mechanisms: $(i)$ the slow capture process ( $s$-process) and $(i i)$ the rapid one ( $r$-process). The $r$-process is thought to occur in high-mass stars, $M \gtrsim 8 M_{\odot}$, exploding as type-II SNe (e.g., Kratz et al. 2007; Travaglio et al. 1999). The $s$-process instead comes from low- and intermediate-mass stars, $1 M_{\odot} \lesssim M \lesssim$ $3 M_{\odot}$ (e.g., Busso et al. 2001), thanks to the repeated neutron expositions guaranteed by the activation of the neutron sources ${ }^{13} \mathrm{C}(\alpha, \mathrm{n}){ }^{16} \mathrm{O}$ and ${ }^{22} \mathrm{Ne}(\alpha, \mathrm{n}){ }^{25} \mathrm{Mg}$. The $s$ process contribution from low-mass stars had a dominant role in the solar mixture of $\mathrm{Ba}$ nuclei, so that in the Sun Ba comes from the s-process at about the $80 \%$ level (Arlandini et al. 1999).

Barium abundances have been measured in stars with different metallicities (e.g., Burris et al. 2000 for halo stars; Cescutti et al. 2006 and Bensby et al. 2005 for thin and thick disk stars), constraining the nucleosynthetic processes at the origin of Ba. However, several details of the $\mathrm{Ba}$ production are still a matter of debate due to the free parameters affecting the models (see, e.g., for the $r$-process Mathews et al. 1992; Pagel \& Tautvaišienè 1997; Travaglio et al. 1999; for the $s$-process Busso et al. 2001). In the $s$-process, the main source of uncertainty is the still unknown physical mechanism promoting the penetration of protons into the He inter-

Electronic address: vdorazi@arcetri.astro.it shell zone at dredge-up; this penetration is necessary for explaining the formation of the main neutron source ${ }^{13} \mathrm{C}$.

In this Letter we address the evolution of $\mathrm{Ba}$ with age by means of Ba measurements in a sample of open clusters (OCs) and by comparing the empirical pattern with the results of a Galactic chemical evolution model. We find an unexpected trend, in apparent conflict with the currently adopted average Ba yields. We also identify in which direction the average yields should be corrected and why this is physically justified.

\section{SAMPLE AND ANALYSIS}

Our sample (see Table 1) consists of 20 OCs spanning a wide interval in age ( $\sim 30 \mathrm{Myr}-8 \mathrm{Gyr})$, Galactocentric radius $(7-22 \mathrm{kpc})$, and metallicity $(-0.3 \leq[\mathrm{Fe} / \mathrm{H}] \leq+0.4)$. We analyzed a large collection of high-resolution spectra obtained by our group during the years with different instruments: UVES in slit-mode and FLAMES/UVES on the ESO VLT, CASPEC on the ESO 3.6m telescope, the Red-Long camera echelle spectrograph at CTIO, and SARG on the Italian National Telescope Galileo. The spectra of unevolved members were analyzed in the first 10 OCs in Table 1, while the analysis of the older and more distant clusters is based on giant stars.

Barium abundances were computed by means of equivalent width (EW) analysis and by using the driver blends in MOOG (Sneden, 1973-2002 version). The grid of 1-D LTE model atmospheres of Kurucz (1993) was employed. Our analysis uses two Ba lines: $\lambda \lambda 5853$ and $6496 \AA$. As well known, Ba lines are affected by hyperfine-splitting (hfs); thus we included in our line list the hyperfine 
structure and isotopic splitting, following the approach by McWilliam (1998) and using log $g f$ values published there. An $81 \% s$-process composition was adopted for the Sun and the sample stars. Stark and radiative broadening were treated in the standard way and for collisional damping we adopted the classical Unsöld (1955) approximation.

As a first step, we derived the solar $\mathrm{Ba}$ abundance using the same method as for the sample stars: for the Sun we adopted $\mathrm{T}_{\text {eff }}=5770 \mathrm{~K}, \log \mathrm{g}=4.44$ and $\xi=1.1 \mathrm{kms} / \mathrm{s}$ (Randich et al. 2006), obtaining $\log \mathrm{n}(\mathrm{Ba})=$ 2.22 and $\log n(\mathrm{Ba})=2.26$ for $\lambda=5853$ and $6496 \AA$, respectively. The average solar value $\log \mathrm{n}(\mathrm{Ba})=2.24 \pm$ 0.02 agrees very well with the meteoritic abundance of $\log n(\mathrm{Ba})=2.22$ (Grevesse et al. 1996). Our analysis is strictly differential with respect to the Sun: we applied a line by line solar correction, canceling out the uncertainties on the adopted set of atomic parameters $(\log g f)$. The feature at $\lambda=6496 \AA$ always yields higher abundances, suggesting, as also stressed by Prochaska et al. (2000), the presence of an unidentified blend; however, the differential nature of our analysis should minimize this effect, in particular for dwarf stars with close-tosolar stellar parameters. Ba abundances were obtained adopting stellar parameters and uncertainties derived in our previous studies.

Errors in Ba abundances for each star are due to uncertainties in EWs and stellar parameters. The standard deviation from the mean of each cluster instead provides a good estimate of the global errors on the average abundances ${ }^{1}$. In order to estimate systematic errors, we compared our results with measurements from the literature for six clusters. We found a good agreement within the uncertainties $(\Delta[\mathrm{Ba} / \mathrm{Fe}]<0.06 \mathrm{dex})$ for M67, Berkeley 20, and NGC 6253; the differences are instead larger for the Hyades $(\Delta[\mathrm{Ba} / \mathrm{Fe}]=-0.2$ dex $)$, Collinder $261(\Delta[\mathrm{Ba} / \mathrm{Fe}]=+0.2 \mathrm{dex})$, and Berkeley 29 $(\Delta[\mathrm{Ba} / \mathrm{Fe}]=+0.14 \mathrm{dex})$. These results show that, whereas discrepancies with the literature are present, these are not systematic; in particular our Ba abundances are not systematically higher. Also, given the old age of $\mathrm{Cr} 261$ and Be 29, our higher [Ba/Fe] ratio for these clusters does not affect our final conclusions.

\section{RESULTS}

The results are summarized in Table 1, where we report the average $[\mathrm{Ba} / \mathrm{Fe}]$ ratios for each cluster along with the standard deviation from the mean. In Figure 1 we show $[\mathrm{Ba} / \mathrm{Fe}]$ ratios as a function of cluster age; different symbols indicate dwarf and giant members. In the figure we also show the solar $[\mathrm{Ba} / \mathrm{Fe}]$ as well as our own measurement for $\alpha$ Centauri performed by using EWs published by Porto de Mello et al. (Porto de Mello et al. 2008). Our $\mathrm{Ba}$ abundance is in excellent agreement with this previous result $(\Delta[\mathrm{Ba} / \mathrm{Fe}]<0.02$ dex $)$. The trend of increasing $[\mathrm{Ba} / \mathrm{Fe}]$ from the oldest clusters to the youngest ones is evident. The figure also shows that $[\mathrm{Ba} / \mathrm{Fe}]$ ratios are systematically higher for clusters where giants have been analyzed. We suggest that this might be due to the fact that our analysis is not strictly differential for gi-

\footnotetext{
1 The line list with the EWs measurements as well as stellar parameters, and $\mathrm{Ba}$ abundances of each single star along with errors are available upon request.
}

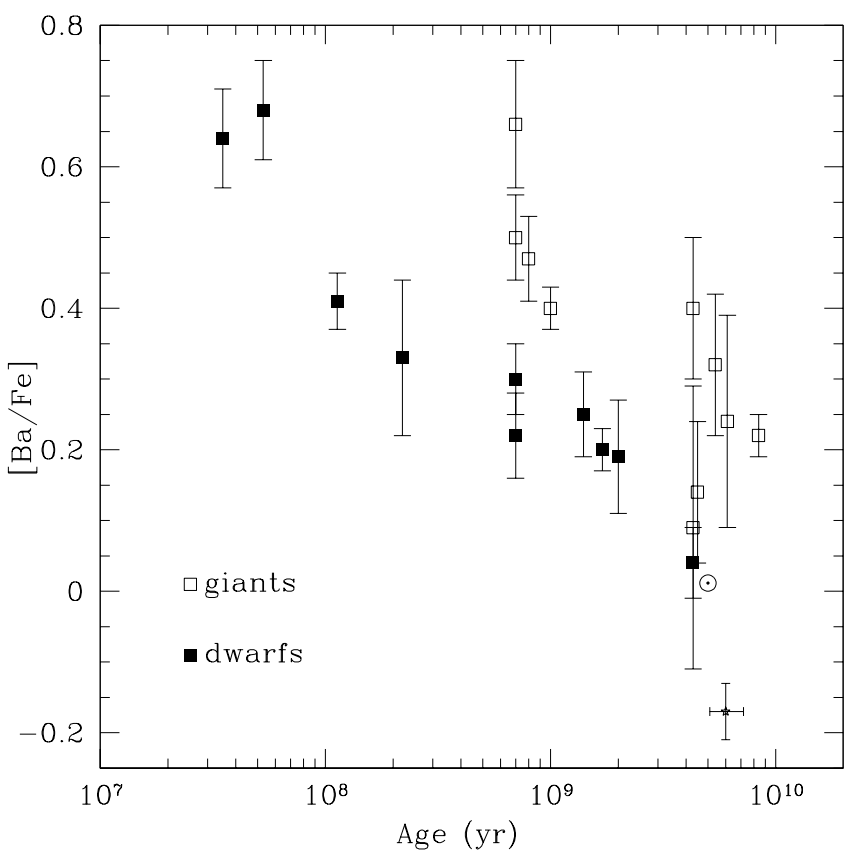

FIG. 1.- Average [Ba/Fe] as function of age for the sample clusters; filled and open squares denote clusters whose analysis is based on dwarf and giant stars, respectively. We also show the solar value (solar symbol) and that of the $\alpha$ Centauri (star with errorbars).

ant stars, as their parameters are different from the solar ones.

First, the question arises whether the observed trend of increasing $[\mathrm{Ba} / \mathrm{Fe}]$ with decreasing age is real or rather due to any effect that, for a given intrinsic Ba abundance, would increase the observed EWs. In particular, members of the youngest clusters in our sample (ages below $\sim 500 \mathrm{Myr}$ ) are characterized by strong magnetic fields and are chromospherically very active; thus Ba II line strengths might be affected by both a "magnetic intensification mechanism" (Landi degl'Innocenti \& Landolfi 2004) and by non-LTE (NLTE) effects. In order to check whether magnetic intensification could be a problem, we measured the EWs of the weak Eu II $6437 \AA$ feature in the sample stars. Magnetic intensification would cause a similar (if not larger) effect for Eu, whose hyper-fine structure is notoriously more complex (the number of Zeeman sub-levels is higher). We found that, at variance with $\mathrm{Ba}, \mathrm{Eu} \mathrm{EWs}$ do not increase with the age, thus excluding that this process affects Ba EWs.

On the other hand, NLTE effects might be important for Ba II features, in particular, for the $\lambda 6496 \AA$ line and for stars with close-to-solar metallicity (Mashonkina et al. 1999). It is very likely that NLTE effects are amplified in young stars due to the presence of hot chromospheres. While we cannot quantify this effect, we believe that $[\mathrm{Ba} / \mathrm{Fe}]$ for clusters younger than $\sim 500 \mathrm{Myr}$ are likely overestimated.

\section{TIME EVOLUTION OF BA}

In Figure 2 we plot the average $[\mathrm{Ba} / \mathrm{Fe}]$ of OCs (only those based on dwarf star analysis) and of field stars by Bensby et al. (2005) vs. stellar age. Although field stars show a larger scatter, the two patterns agree very well and our OCs do extend the same trend to younger ages. 


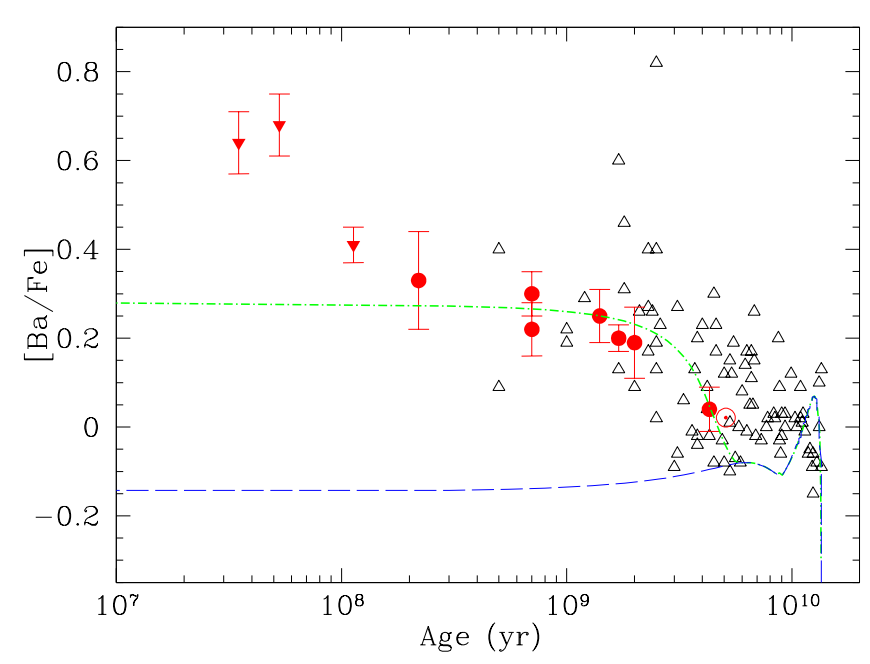

FIG. 2.- Average $[\mathrm{Ba} / \mathrm{Fe}]$ as function of stellar age for the subsample of clusters whose analysis is based on dwarfs (filled circles and inverted triangles) compared with the abundance pattern of disk stars (open triangles) by Bensby et al. (2005). Filled triangles represent abundance measurements that probably need NLTE corrections. The model results are shown for two set of yields: (a) standard yields (Travaglio et al. 1999; Busso et al. 2001), longdashed curve; and $(b)$ enhanced $s$-process yields, dot-dashed curve. Both models show a peak at old ages due to the $r$-process from massive stars.

Qualitatively, the observed trend of Ba vs. age confirms the dominant role played, in Galactic disk stars, by the $s$-process occurring in low-mass red giants during their asymptotic giant branch (AGB) phase (Busso et al. 2001; Cescutti et al. 2006). For a more quantitative comparison, we also show in Fig. 2 the time evolution of $[\mathrm{Ba} / \mathrm{Fe}]$ computed with a Galactic chemical evolution model for the Milky Way. The model adopted is a generalization of the multi-phase model by Ferrini et al. (1992), originally built for the solar neighborhood, and later extended to model the entire Galaxy (Ferrini et al. 1994). We refer to Magrini et al. (2007, 2008) for a detailed description of the model. In our "standard" model we adopted the average $s$-process and $r$-process yields by Busso et al. (2001) (extrapolated as in Cescutti et al. 2006) and Travaglio et al. (2001), respectively. While the $r$-process yields do not affect the $[\mathrm{Ba} / \mathrm{Fe}]$ ratio in the metallicity range of our OCs (the $r$-process is important only for $[\mathrm{Fe} / \mathrm{H}] \lesssim-2.2$ ), the values of the $s$-process yields are crucial to reproduce the increasing trend of $[\mathrm{Ba} / \mathrm{Fe}]$ with decreasing age of the cluster.

The figure clearly shows that the model with standard yields does not reproduce the empirical distribution, and, in particular the observational evidence that at recent epochs $\mathrm{Ba}$ is sensitively enhanced both for field stars and for OCs. The standard $s$-process yields do not even fit the solar $[\mathrm{Ba} / \mathrm{Fe}]$ value and result in a $\mathrm{Ba}$ abundance ratio $[\mathrm{Ba} / \mathrm{Fe}] \approx-0.15$ at $1 \mathrm{Gyr}$, to be compared with the measured value of $\sim 0.2$. In the original work from where the $s$-process yields were taken (Busso et al. 2001 ) it was underlined that at every epoch and for every group of stars there was evidence of a large spread in their efficiencies. However, a simply randomly distributed spread seems not to be the case: it would generate from each given stellar generation a yield close to the average one adopted by us, and this is clearly inadequate to explain the data. Instead, we "empirically" veri- fied that the observations, showing a very late increase of $\mathrm{Ba}$ in the Galaxy, would require an efficiency of production strongly weighted toward lower masses. Namely, the observations can be well fitted by increasing the yields of stellar masses between 1 and $1.5 M_{\odot}$ by a factor of $\sim 6$ with respect to the extrapolation obtained from the average yields (roughly corresponding to the case ST/1.5 in Busso et al. 2001).

The results of a chemical evolution model where we have adopted "enhanced $s$-process" yields are shown in Fig. 2. In this model the solar Ba abundance can be reproduced, with contributions from the $s$ - and $r$-process of $84 \%$ and $16 \%$, respectively. As mentioned, the model with standard yields has instead a lower percentage of Ba produced by the $s$-process at the time of formation of the Sun. More in general, the model with enhanced yields provides a recent production of $\mathrm{Ba}$ in better agreement with the observations of OCs older than $\sim 500 \mathrm{Myr}$. Both models fail to fit younger clusters and, indeed, it would be difficult to imagine a process capable of creating $\mathrm{Ba}$ in the last $500 \mathrm{Myr}$ of Galactic evolution, unless local enrichment is assumed. We suggest that the difference between our model predictions and the measured $[\mathrm{Ba} / \mathrm{Fe}]$ ratios for young clusters provides an estimate of the NLTE effects that affect the Ba determination in these stars.

We finally stress that the classical plots of $[\mathrm{Ba} / \mathrm{Fe}]$ against $[\mathrm{Fe} / \mathrm{H}]$ (see, e.g., Travaglio et al. 1999, 2001; Cescutti et al. 2006) suffer for a strong degeneration in terms of age, since they compress the most recent period. Conversely, the plot of $[\mathrm{Ba} / \mathrm{Fe}]$ against age favors the youngest ages and thus is ideal to approach our problem. We also investigated the presence of a possible relationship of $[\mathrm{Ba} / \mathrm{Fe}]$ with metallicity among our sample, finding that no significant trend is present between these two quantities.

\section{DISCUSSION}

Our results show for the first time there is a need for an enhanced $\mathrm{Ba}$ production in low-mass stars, mainly from 1 to $1.5 M_{\odot}$, formed from $\sim 10$ to $\sim 5$ Gyr ago (e.g. Charbonnel et al. 1996). We have shown that, when adopting the average yields that have been commonly used so far, Galactic evolutionary models are in sharp disagreement with data. An enhanced Ba production, like the one assumed in the previous section, seems to be necessary, but one has to verify whether this ad-hoc data-fitting is physically acceptable.

The most promising possibility to obtain enhancements in the Ba production, especially in low masses, is that the intrinsic spread found normally in the $s$-process efficiency at any metallicity be not really a spread, but rather a dependence of the efficiency itself on the initial mass, in the sense of higher production for lower masses. As time passes, lower mass stars with larger Ba production start to contribute, and the average yield changes in time. Specifically, the formation of the neutron source ${ }^{13} \mathrm{C}$ requires a penetration of protons below the formal convective border at dredge-up. This is a typical extramixing process, like those necessary to account for the carbon and oxygen isotopic mix in low-mass red giants, and for the production and destruction of $\mathrm{Li}$ in them. These mechanisms are known to be increasingly more efficient for decreasing stellar mass. The evidence com- 
ing from the $\mathrm{Ba}$ enhancement indirectly suggests that the mixing processes driving the formation of the neutron source behave similarly, and are maybe originated by the same cause (e.g. Busso et al. 2007). In general, we verified that the efficiency in $s$-processing required in very low mass stars in order to explain the data is close to the upper limit (case $\mathrm{ST}^{*} 2$ ) suggested by Busso et al (2001), not to the average of the spread there considered (case ST/1.5). This must come together with the (already known) increase in the dredge-up efficiency at low metallicity, so that this large amount of extra Ba can be carried to the surface and ejected into the ISM.

Other possibilities were also considered such as: $i$ ) an increase in the initial $s$-element abundances of the contributing stars, due to previous enrichment by mass transfer from more massive companions; and ii) an increase in the $r$-process seeds on which slow n-captures occur, assuming that old disk stars were still characterized by high $r$-process abundances. Both attempts proved incapable of increasing substantially the Ba yields.

\section{CONCLUSIONS}

High-resolution observations of OCs allowed us to derive $\mathrm{Ba}$ abundances in a uniform way in systems covering a wide age range. The $[\mathrm{Ba} / \mathrm{Fe}]$ derived in OCs (but also in field stars, by other authors) shows a steady increase with time. In the framework of Galactic chemical evolution, this can be explained by an enhanced $s$-process production of $\mathrm{Ba}$ in very old AGB stars below $1.5 \mathrm{M}_{\odot}$.

This need can be reconciled with the present understanding of the $s$-process if an anti-correlation exists between the initial mass of the star and the effectiveness of the extra-mixing mechanism responsible for forming the neutron source. Stars at the lowest mass limit for contributing would in this case have a higher efficiency in $s$-processing. Accounting for the observations then requires that this efficiency be close to the upper limit indicated by Busso et al. (2001), i.e. an amount of ${ }^{13} \mathrm{C}$ burnt per interpulse higher by a factor of three than the gross average (case ST/1.5) discussed in that work: this would produce an increase in the Ba yield by a factor of 5-6. This scenario is plausible if the mechanisms driving the formation of the ${ }^{13} \mathrm{C}$ pocket are similar to those promoting the slow circulations known to occur below the convective envelope.

The youngest clusters of our sample, with ages < $500 \mathrm{Myr}$, have even higher $[\mathrm{Ba} / \mathrm{Fe}]$ ratios. We suggest that this is due to NLTE effects, and, in particular, to the effects of a strong, warm flux from a chromosphere. The comparison with our model suggests that NLTE effects in young stars might increase the $[\mathrm{Ba} / \mathrm{Fe}]$ ratios by a factor of two of more. Independent estimates of these effects from NLTE calculations as well as a more complete analysis of the hypothesis here advanced of an anticorrelation between initial mass and $s$-process efficiency are strongly needed.

We are grateful to A. McWilliam and C. Sneden for many helpful suggestions. We warmly thank L. Belluzzi and G. Cauzzi for useful discussions. The anonymous referee is acknowledged for a careful reading of the paper and valuable comments. This work has made use of the WEBDA database, originally developed by J. C. Mermilliod, and now maintained by E. Paunzen.

\section{REFERENCES}

Arlandini, C., Käppler, F., Wisshak, K. 1999, ApJ, 525, 886 Bensby, T. Feltzing, S., Lundström, Ilyin, I. 2005, A\&A, 433, 185 Bragaglia A., Sestito P., Villanova, S., Carretta, E., Randich, S., Tosi, M. 2008, A\&A, 480, 79

Busso, M., Gallino, R., Lambert, D.L., Travaglio, C., \& Smith, V.V. 2001, ApJ, 557, 802

Busso, M., Wasserburg, G.J., Nollet, K.M., Calandra, A. 2007, ApJ, 671, 802

Burris, D. L., Pilachowski, C. A., et al. 2000 ApJ, 544, 302 Cescutti, G., François, P., Matteucci, F., Cayrel, R., Spite, M. 2006, A\&A, 448, 557

Charbonnel, C., Meynet, G., Maeder, A., Schaerer, D. 1996, A\&AS, 115, 339

D’Orazi, V., Randich, S. 2008, A\&A, submitted

Ferrini, F., Matteucci, F., Pardi, C., Penco, U. 1992, ApJ, 387, 138

Ferrini, F., Mollà, M., Pardi, M. C., Diaz, A. I. 1994, ApJ, 427, 745

Grevesse, N., Noels, A., Sauval, A. J. 1996, ASP Conf. Ser. 99, 117

Kratz, K-L., Farouqi, K., Mashonkina, L.I., and Pfeiffer, B. 2007, New Astr. Rev. 52, 390

Kurucz, R.L., 1993, CD-ROM Nr. 9

Landi degl'Innocenti, E., Landolfi, M. 2004, Polarization in spectral lines, Kluwer Academic Publishers

Magrini, L., Corbelli, E., Galli, D. 2007, A\&A, 470, 843

Magrini, L., Sestito, P., Randich, S., Galli, D. 2008, A\&A, in press (arXiv:0812.0854)

Mashonkina, L., Gehren, T., Bikmaev, I. 1999, A\&A, 343, 519
Mathews, G.J., Bazan, G., Cowan, J.J. 1992, ApJ, 391, 719

McWilliam, A., 1998, AJ, 115, 1640

Pace, G., Pasquini, L., François, P. 2008, A\&A, 489, 403

Pagel. B.E.J., Tautvaišienè, G. 1997, MNRAS, 288, 108

Paulson, D.B., Sneden, C., Cochran, W.D. 2003, AJ, 125, 3185

Porto de Mello, G.F., Lyra, W., Keller, G.R. 2008, A\&A, 488, 653

Prochaska, J.X., Naumov, S.O., Carney, B.W., McWilliam, A., Wolfe, A.M. 2000, AJ, 120, 2513

Randich, S., Sestito, P., Primas, F., Pallavicini, R., Pasquini, L. 2006, A\&A, 450, 557

Randich, S., Primas, F., Pasquini, L., Sestito, P., Pallavicini, R. 2007, A\&A, 469, 163

Sestito, P., Randich, S., Mermilliod, J.C., Pallavicini, R. 2003, A\&A, 407, 289

Sestito, P., Randich S., Pallavicini, R. 2004, A\&A, 426, 809

Sestito, P., Randich, S. 2005, A\&A, 442, 615

Sestito, P., Bragaglia, A., Randich, S., Carretta, E., Prisinzano, L., Tosi, M. 2006, A\&A, 458, 121

Sestito, P., Randich, S., Bragaglia, A. 2007, A\&A, 465, 185S

Sneden, C.A. 1973, ApJ, 184, 839

Terndrup, D.M., Pinsonneault, M., Jeffries, R.D., et al. 2002, ApJ, 576, 950

Travaglio, C., Galli, D., Gallino, R., et al. 1999, ApJ, 521, 691

Travaglio, C., Galli, D., Burkert, A. 2001, ApJ, 547, 217

Unsöld, A., 1955, Physik der Sternatmosphären (Berlin:

Springer-Verlag) 
TABLE 1

OC SAMPLE

\begin{tabular}{lrrrrrr}
\hline Cluster & Age $(\mathrm{Gyr})$ & {$[\mathrm{Fe} / \mathrm{H}]$} & Ref. & {$[\mathrm{Ba} / \mathrm{Fe}]$} & $\#$ stars & $T_{\text {eff }}(\mathrm{K})$ \\
IC 2602 & 0.035 & $0 \pm 0.01$ & 1 & $0.64 \pm 0.07$ & 8 & $4770-5760$ \\
IC 2391 & 0.053 & $-0.01 \pm 0.02$ & 1 & $0.68 \pm 0.07$ & 6 & $4680-5970$ \\
NGC 2516 & 0.110 & $0.01 \pm 0.07^{\mathrm{a}}$ & 2 & $0.41 \pm 0.04$ & 4 & $5110-5659$ \\
NGC 6475 & 0.22 & $0.14 \pm 0.06$ & 3 & $0.33 \pm 0.11$ & 19 & $5048-5888$ \\
Hyades & 0.7 & $0.13 \pm 0.05^{\mathrm{b}}$ & 2 & $0.30 \pm 0.05$ & 4 & $5079-5339$ \\
Praesepe & 0.7 & $0.27 \pm 0.04$ & 4 & $0.22 \pm 0.06$ & 6 & $5720-6280$ \\
NGC 3680 & 1.4 & $-0.04 \pm 0.03$ & 4 & $0.25 \pm 0.06$ & 2 & $6010-6210$ \\
IC 4651 & 1.7 & $0.12 \pm 0.05$ & 4 & $0.20 \pm 0.03$ & 5 & $5910-6320$ \\
NGC 752 & 2.0 & $0.01 \pm 0.04$ & 5 & $0.19 \pm 0.08$ & 9 & $5531-6151$ \\
M67 & 4.3 & $0.02 \pm 0.14$ & 6 & $0.04 \pm 0.05$ & 10 & $5541-6223$ \\
& & & & & & \\
\hline
\end{tabular}

Note. - Results of [Ba/Fe] ratios for our sample OCs. We list: ages (Col. 2) from Magrini et al. (2008), with the exception of NGC 2516 (WEBDA) and NGC 752 (Sestito et al. 2004); [Fe/H] values along with references for them and stellar parameters (Cols. 3 and 4); average $[\mathrm{Ba} / \mathrm{Fe}]$ ratios along with the standard deviation (Col. 5); number of stars and range of effective temperatures (Cols. 6 and 7 ). Reference codes are as follows: $1=$ D'Orazi \& Randich (2008), $2=$ Randich et al. (2007), $3=$ Sestito et al. (2003), 4=Pace et al. (2008), 5=Sestito et al. (2004),6=Randich et al. (2006), 7=Bragaglia et al. (2008), 8=Sestito et al. (2006), 9=Sestito et al. (2007), 10=Sestito et al. (2008).

aTerndrup et al. (2002)

${ }^{b}$ Paulson et al. (2003) 